\title{
Effect of ivermectin on infection with gastro-intestinal nematodes in Sierra Leone
}

\author{
J.M. Behnke ${ }^{1}$, D.I. Pritchard', D. Wakelin ${ }^{1}$, J.R. Park ${ }^{1}$, \\ A.M. McNicholas ${ }^{2}$ and F.S. Gilbert ${ }^{3}$ \\ ${ }^{1}$ MRC Experimental Parasitology Research Group and ${ }^{3}$ Behaviour and \\ Ecology Research Group, Department of Life Science, University of \\ Nottingham, University Park, Nottingham NG7 2RD, UK \\ ${ }^{2}$ MRC Laboratories, P.O. Box 81 , Bo, Sierra Leone
}

\begin{abstract}
Levels of intestinal nematode infections were assessed six months after a two year trial of ivermectin for efficacy against onchocerciasis had ended. In the trial the inhabitants of six villages in Sierra Leone were offered treatment with ivermectin or placebo at six monthly intervals for four rounds in total. Quantitative faecal egg counts were carried out on stool samples provided by 202 subjects, all of whom had received all four rounds of treatment, in two of the villages (Dodo and Mogibisi). These data were analysed by a novel procedure in which a 3-way ANOVA with negative binomial errors enabled village and host gender influences on the outcome of treatment to be identified. Necator americanus was the most common species showing an overall prevalence of $90 \%$ but a higher intensity in Mogibisi relative to Dodo, particularly among male subjects. Neither prevalence nor intensity of infection were altered in ivermectin-treated compared with placebo-treated subjects. Trichuris trichiura was the least common species with a prevalence of $15 \%$. It was concluded that there was no significant protection from infection with this species among the ivermectin relative to the placebo-treated subjects. Ascaris lumbricoides, with an overall prevalence of $39 \%$, was more common among female $(50 \%)$ compared with male $(27 \%)$ villagers. Prevalence was not significantly affected by ivermectin but the intensity of infection declined by $91.3 \%$ in Dodo whilst in Mogibisi intensity was only reduced by $14.6 \%$. The results indicate that the long-term benefits of treatment of necatoriasis and trichuriasis with ivermectin are likely to be minimal whilst those of ascariasis will depend on the individual characteristics of local communities and may differ quite markedly within a limited geographical area. Possible explanations for the discrepancy in efficacy between villages are considered.
\end{abstract}

\section{Introduction}

The anthelmintic ivermectin has a broad spectrum of activity against parasitic nematodes, and is widely used for the control of gastrointestinal species in domestic animals (Benz et al., 1989). Although it is currently used in man only against Onchocerca volvulus, a number of reports have confirmed that the drug is also active against intestinal nematodes (Campbell, 1985; Whitworth et al., 1991). The regular use of ivermectin in onchocerciasis control programmes may therefore confer the additional benefit of reducing burdens of intestinal nematode infections. However, ivermectin is not equally effective against the major gastrointestinal nematodes Ascaris, hookworms and Trichuris (Richard-Lenoble et al., 1988; Whitworth et al., 1991; Behnke et al., 1993), and it is well established that infections with these species in the community can show considerable resilience during and post chemotherapy, 
intensities returning to pretreatment values relatively rapidly (Seo et al., 1980; Bundy et al., 1985; Quinnell et al. 1993)

The implementation of a long-term, placebo-controlled programme of ivermectin treatment in the Bo region of Sierra Leone has provided the opportunity to monitor long term effects of treatment on the levels of gastrointestinal nematode infection in the population. Preliminary, semiquantitative analyses of these infections have been reported by Whitworth et al. (1991). The data described here are based on detailed quantitative analyses of infections with Ascaris lumbricoides, Necator americanus and Trichuris trichiura in 202 individuals from two villages. However, in contrast to earlier studies, rather than merely compare placebo and treated groups directly as is often the case in chemotherapy trials of this type, we considered it possible that the outcome of treatment might differ between the villages and host genders. In order to facilitate appropriate statistical analysis, a novel procedure is described, in which a 3-way ANOVA with negative binomial errors allowed the influences of village and host gender on the outcome of treatment to be identified. This procedure is given in detail since it is likely to prove useful for the analysis of overdispersed data-sets from other surveys.

\section{Materials and methods}

\section{Study site and design}

A double-blind placebo-controlled trial of ivermectin was initiated in 1987 in six villages with hyperendemic onchocerciasis along the Tabe river in southeastern central Sierra Leone near the city of Bo. Individuals were randomized to receive ivermectin ( $150 \mu \mathrm{g} / \mathrm{kg}$ body weight) or identical placebo. Children under 5 years, pregnant women, lactating women with babies less than 1 month old, villagers with neurological disease or severe systemic illness were excluded from treatment with ivermectin in accordance with the manufacturer's guide lines. The original design for this study did not include an assessment of the effects of ivermectin against GI nematodes, since the principal objective was to assess the value of ivermectin in the treatment of onchocerciasis and therefore background data were not collected on the intensity of infections among the participating subjects prior to treatment. However, the need to quantify the effects of ivermectin arose when it became apparent, after the first rounds of treatment, that some individuals continued to pass ascarids in large numbers with each successive round indicating that long-term protection was unlikely to be afforded by this anthelmintic. The study design and initial observations on changes in GI nematodes following treatment as well as reinfection rates were reported by Whitworth et al. (1991). The present study is based on the inhabitants of two of the participating villages (Dodo and Mogibisi) and included all available individuals in these villages who had received all four rounds of earlier treatment with ivermectin or placebo.

\section{Collection of stools and qualification of GI infections}

Samples of overnight stools were collected from 202 individuals ( 143 from Dodo and 59 from Mogibisi) in March 1990 and each sample was classified into one of five categories; hard-formed, formed, soft-formed, mushy or diarrhoeic, according to WHO (1963) guidelines. Eggs were counted by the formol-ether technique on samples of known weight in which the sediment containing eggs was resuspended in a known volume of formalin $(1.25 \mathrm{ml})$. Three $10 \mu \mathrm{l}$ samples were counted under a microscope and all negative samples were recounted at least a further three times, as were samples in which initial counts varied excessively. Egg counts were then expressed as eggs per gram of faeces (EPG).

Fifty two individuals from Dodo were treated with Combantrin (Pfizer Ltd., Sandwich, Kent) at the recommended dose for age (approximately $10 \mathrm{mg} / \mathrm{kg}$ body weight; Pritchard et al., 1990). Stool samples from these individuals were collected 24 and $48 \mathrm{~h}$ after treatment and worms were isolated after washing faecal material through a combination of two sieves (mesh sizes $1 \mathrm{~mm}$ and $300 \mu \mathrm{m}$ ) with local river water, at a site specially prepared for this task at some distance from the village. Worms were isolated, fixed in formalin and identified. All the hookworms recovered were $N$. americanus. Subsequently all the remaining individuals participating in the study were offered free treatment with Combantrin.

\section{Statistical analysis of results}

Prevalence data were examined using log-linear models (Sokal \& Rohlf, 1981) implemented by Statgraphics v. 2.6, command Loglin. These models fit the cell frequencies to an explicit model: in our case the model was

$$
\operatorname{Ln}\left(f_{i j k k}\right)=\mu+\alpha_{i}+\beta_{i}+\gamma_{k}+\delta_{1}+\alpha \beta_{i j}+\alpha \gamma_{i k} \ldots \alpha \beta \gamma \delta_{i j k l}
$$

where $f_{i j k d}$ is the expected frequency in village $i$, sex $j$, treatment $k$ and prevalence 1 . Because there were more individuals sampled from one village (Dodo), there should always be significant coefficients for the village effect $(\alpha)$. Similarly, prevalence is never $50 \%$ and so significant coefficients for the overall effect of prevalence $(\delta)$ should be expected. For each variable the highest order effect from the full 4-way interaction (infected/uninfected $\times$ gender $\times$ village $\times$ treatment) down to the simple main effects was tested using the option 'test order'. The appropriate model was then fitted and checked for its adequacy, deleting components successively to arrive at a minimal adequate model. Values quoted in the text are for the log-likelihood ratio, distributed as $\chi^{2}$, for the difference between the fitted model and the actual data.

Data for faecal egg counts were analysed as raw values for eggs per gram of faeces or after adjustment using WHO (1963) guidelines as follows: formed $\times 1$, hard-formed $\times 0.5$, soft-formed $\times 1.5$, mushy $\times 2$, diarrhoeic $\times 3$. Mean EPG were then calculated on unadjusted and adjusted data for each species of parasite by treatment, and by village, gender, and village and gender. Data were analysed by the statistical package GLIM (Numerical Algorithms Group) on the University of Nottingham Mainframe ICL computer. Worm burdens are known to be distributed according to the negative binomial distribution (Keymer \& Pagel, 1990). The data were overdispersed since the negative binomial constant $k$, calculated for the adjusted values in the entire data-set $(n=202)$ was 0.392 for $N$. americanus, 0.091 for $A$. lumbricoides and 0.068 for $T$. trichiura. The design of the analysis was a three-way ANOVA (village, sex, treatment) 


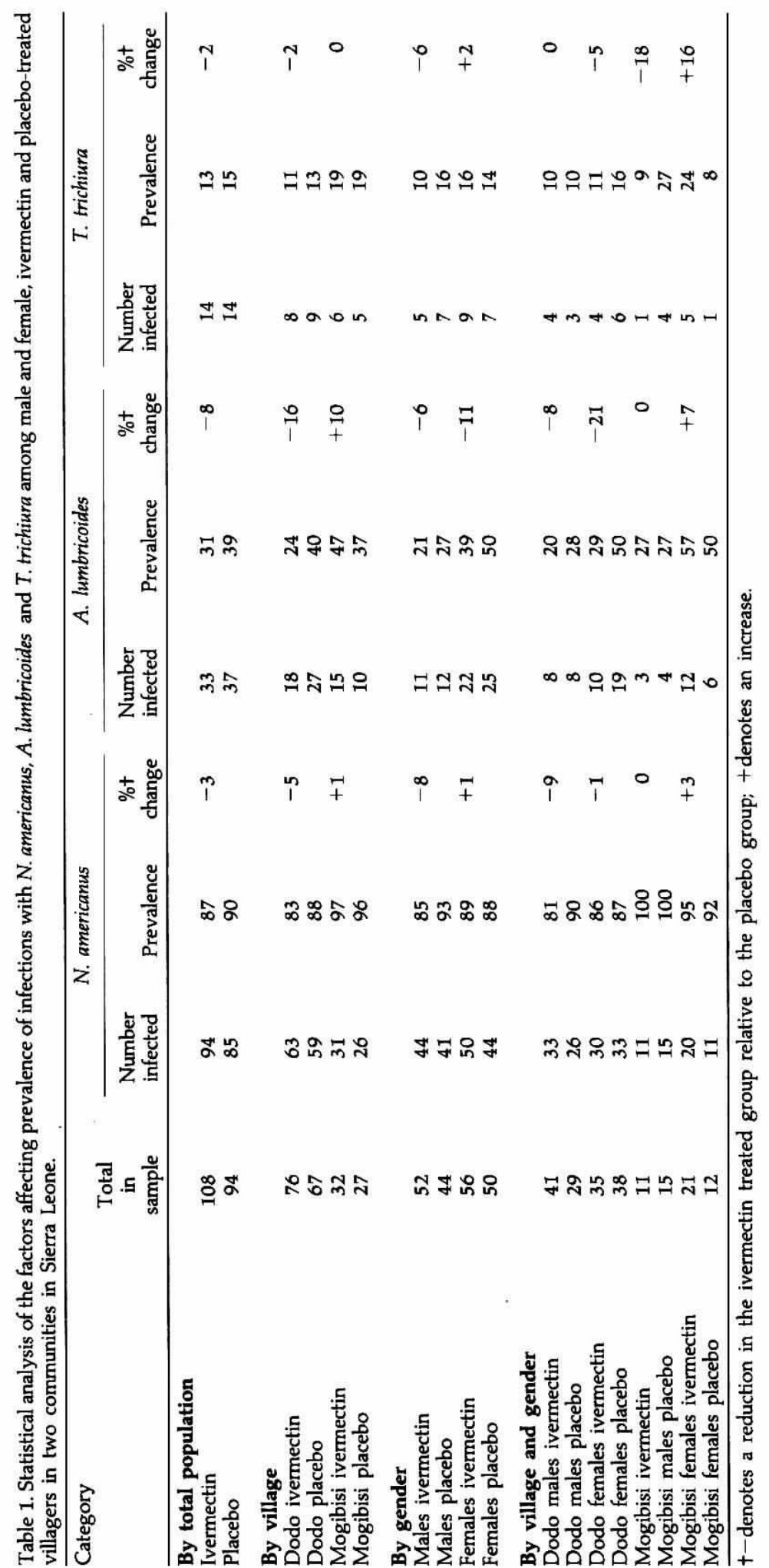


with negative binomial errors. This was analysed for each species using GLIM with a log-link function (see appendix), kindly supplied by Dr C. West (Department of Public Health, University of Liverpool). For each parasite, the adjusted EPG values were used in the full factorial model, and then terms were deleted successively, starting with the highest order interaction, to obtain the changes in deviance and degrees of freedom. The change in deviance divided by the scale parameter represents the log-likelihood ratio for that component in the model and is distributed as $\chi^{2}$.
1, most ivermectin and placebo-treated groups were numerically balanced, with the exception of three groups; males in Dodo (41:29), males in Mogibisi (11:15) and females in Mogibisi (21:12).

\section{Necator americanus}

Prevalence in the combined placebo groups (table 1) was $90 \%$. It was numerically higher in Mogibisi than in Dodo and slightly higher in males than in females. The largest

\section{APPENDIX}

Calculating an ANOVA with negative binomial errors. The following is a GLIM macro that implements negative binomial errors, kindly supplied by Dr. Chris West (Liverpool University).

The data were overdispersed, and therefore we used a log-link function, as follows:

\begin{tabular}{|c|c|c|c|}
\hline $\begin{array}{l}\text { \$MAC } \\
\text { \$MAC } \\
\text { \$MAC } \\
\text { \$MAC } \\
\text { \$MAC } \\
\text { \$MAC } \\
\text { \$MAC }\end{array}$ & $\begin{array}{l}\text { NB1 \$CAL } \\
\text { NB2 \$CAL } \\
\text { NB3 \$CAL } \\
\text { NB4 \$CAL } \\
\text { NB4W \$CAL } \\
\text { INIT \$CAL } \\
\text { CHK } \\
\text { \$CAL } \\
\text { PRINT }\end{array}$ & 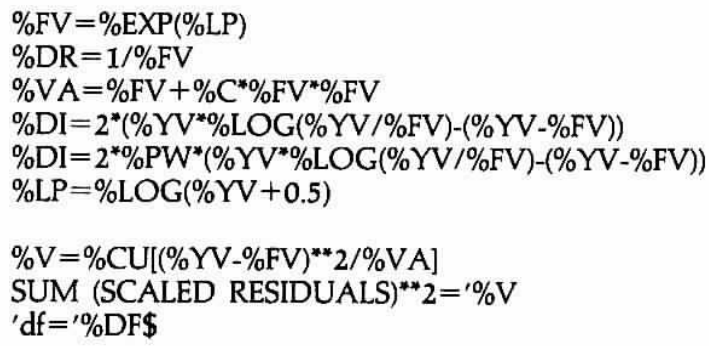 & $\begin{array}{l}\text { \$ENDM } \\
\text { \$ENDM } \\
\text { \$ENDM } \\
\text { \$ENDM } \\
\text { \$ENDM } \\
\text { \$ENDM }\end{array}$ \\
\hline
\end{tabular}

\section{\$ENDM}

\$OWN

$$
\text { NB1 NB2 NB3 NB4 }
$$

Then we used \$SCALE 1 , fitted the full factorial model, and used \$CAL \%C=a range of values until the sum of the scaled residuals (obtained using \$USE CHK) was approximately equal to the degrees of freedom. When this is true, the S.E.M.s of the parameters are approximately correct.

Having obtained the full factorial model, we then removed single components and all others containing it, and noted down the changes in deviance and degrees of freedom (df). The change in deviance was divided by the scale parameter to give the log-likelihood ratio, measuring the extent to which that removed component accounted for the variation in the data.

\section{Results}

Effect of ivermectin on the prevalence of infection

Possible changes in the prevalence of infection after treatment with ivermectin were examined at the levels of (a) the combined populations, (b) the populations in each village, (c) males and females in the combined populations and (d) males and females in each village. In each case the ivermectin-treated group was compared directly to its equivalent placebo-treated group, As can be seen from table discrepancy between placebo and treated groups was among the male population of Dodo (ivermectin $81 \%$ versus placebo 90\%). However, log-linear analysis (table 2) suggested that there were no interactions between factors, and that a simple model including only the effects of village and prevalence was sufficient to account for the data ( $L=16.413$, 13d.f., $P=0.23$ ). The actual coefficients could not be estimated because there were empty cells as a consequence of $100 \%$ prevalence of hookworms among the male subjects in Mogibisi.

Table 2. Statistical analysis of the factors affecting prevalence of infections with $N$. americanus, A. lumbricoides and T. trichiura among male and female, ivermectin and placebo-treated villagers in two communities in Sierra Leone.

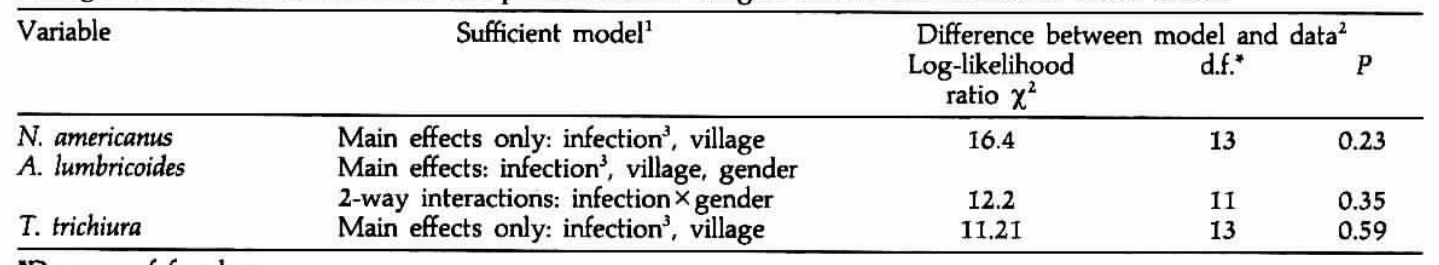

"Degrees of freedom.

${ }^{1}$ Significant components of the factorial ANOVA.

${ }^{2}$ Test of whether the sufficient model in Column 2 accounts for the data. A non-significant probability indicates that no other factors apart from those specified contribute significantly to the observed pattern of the data.

${ }^{3}$ Prevalence of infection. 
Table 3. Statistical analysis of the factors affecting the intensity of infections with $N$. americanus, A. lumbricoides and T. trichiura among male and female, ivermectin-treated and placebo-treated villagers in two communities in Sierra Leone.

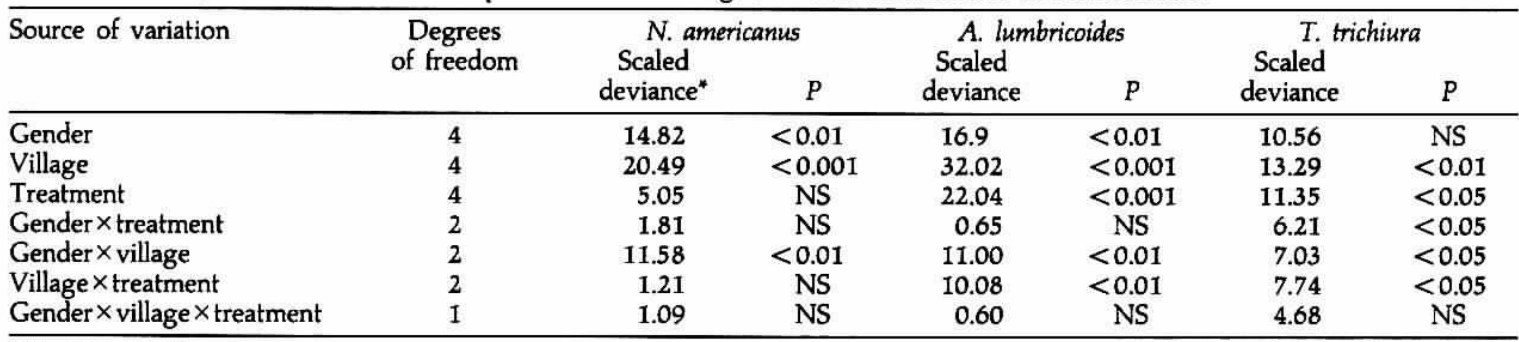

NS $=$ Not significant.

"Scaled deviance is a measure of the contribution of the factor specified under the column labelled 'source of variation' to explaining the variation in the data. It is calculated by fitting an analysis of variance with negative binomal errors through GLIM and is distributed as $\chi^{2}$. For full details see Materials and Methods and Appendix 1.

Ascaris lumbricoides

Ascaris infections were less common than hookworms (table 1), with an overall prevalence in the placebo group of $39 \%$. There was little difference between the two villages (Dodo, $40 \%$ versus Mogibisi, $37 \%$ ). However, there was a marked difference between the sexes (males $27 \%$, females $50 \%$ ), and this was similar in both villages (Dodo males $28 \%$, females $50 \%$; Mogibisi, males $\mathbf{2 7} \%$, females $50 \%$ ). Treatment with ivermectin had a relatively small effect on overall prevalence $(8 \%$ reduction, combined sexes; males $6 \%$ and females $11 \%$ ). The overall prevalence of $A$. lumbricoides was $16 \%$ lower in the treated group in Dodo (males by $8 \%$, females by $21 \%$ ), but $10 \%$ higher among treated persons in Mogibisi. However, the log-linear analysis (table 2) showed that there was no significant treatment $\times$ village interaction, and a model containing prevalence, gender, village and the interaction, prevalence $\times$ gender, accounted for the data.

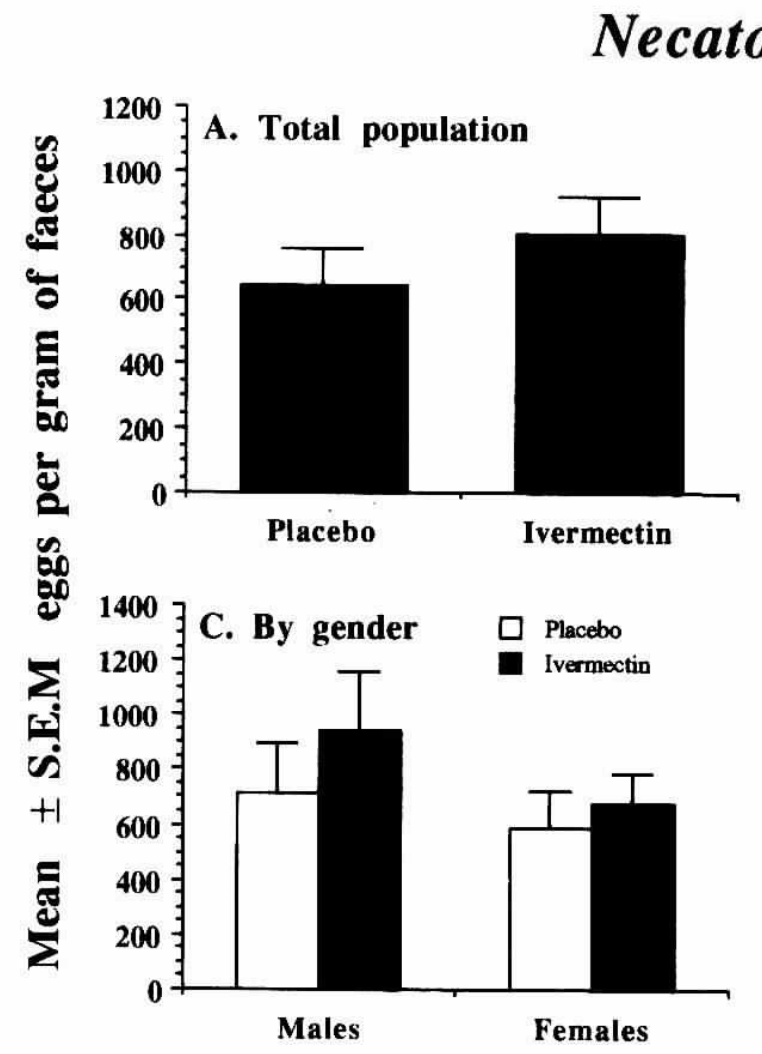

Fig. 1. Intensity of infections with Necator americanus in ivermectin-treated and placebo-treated subjects in the total population, by village and gender.
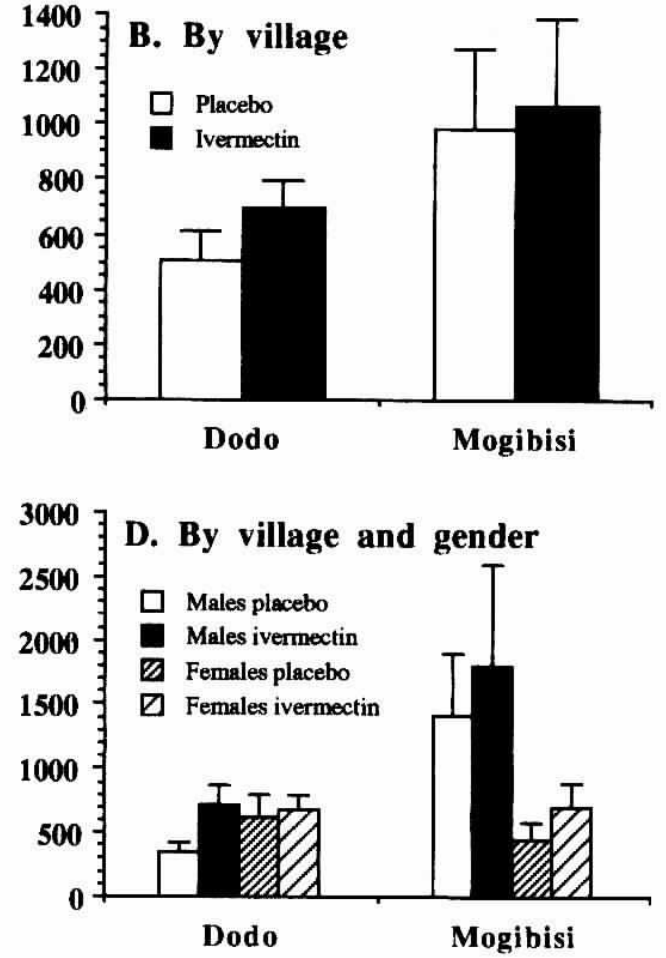
Thus treatment with ivermectin did not have a significant effect on prevalence, but prevalence was influenced by gender.

\section{Trichuris trichiura}

This was the rarest of the three GI nematodes monitored (table 1). Overall prevalence was $15 \%$ in the combined placebo group, slightly higher in Mogibisi compared with Dodo, and similar between the sexes. Log-linear analysis (table 2) showed that a model including only prevalence and village as main effects was adequate to account for the data. Thus ivermectin did not influence the prevalence of infection and prevalence was not dependent on host gender. Curiously, the data for the outcome of treatment on prevalence in Mogibisi were consistent with the remaining categories in that, when tabulated by gender and treatment (table 1), prevalence was $18 \%$ lower among males and $16 \%$ higher among females, thus cancelling each other out when considered at the total village level (placebo and ivermectin both $19 \%$ prevalence). However, these changes were based on small sample sizes and were not statistically significant.

Effect of ivermectin on the mean intensity of infection

The intensity of infection was assessed by faecal egg counts and the data were analysed as raw counts (EPGs) and after adjustment of individual raw data, for stool consistency. The statistical analysis in table 3 is based only on adjusted values. Of the 202 subjects $146(72.3 \%)$ had formed stools and their EPG values were therefore not changed. Five subjects had diarrhoeic stools, 27 subjects had mushy stools, 17 had soft-formed stools and 7 had hard-formed stools.

Both raw and adjusted EPGs for each species, whether treated with ivermectin or placebo were distributed according to a negative binomial distribution. As expected, the species showing the lowest prevalence and placebo-treated subjects ( $T$. trichiura) had the most aggregated distribution with the lowest value of $k(0.12)$. N. americanus had the highest $k$ value $(0.41)$ and accordingly the highest prevalence.

\section{Necator americanus}

The mean intensity of infection with $N$. americanus (fig. 1), as reflected in adjusted eggs per gram of faeces (EPG), was almost twice as high in Mogibisi compared with Dodo (fig. 1B, 986 vs 506 respectively). Overall, the mean values among placebo males and females were similar (fig. 1C) but there was a significant gender $\times$ village interaction (table 3, $P<0.01$ ). EPGs among placebo-treated female subjects were very similar in the two villages (Dodo 625; Mogibisi, 451) but there was a pronounced difference

\section{Ascaris lumbricoides}
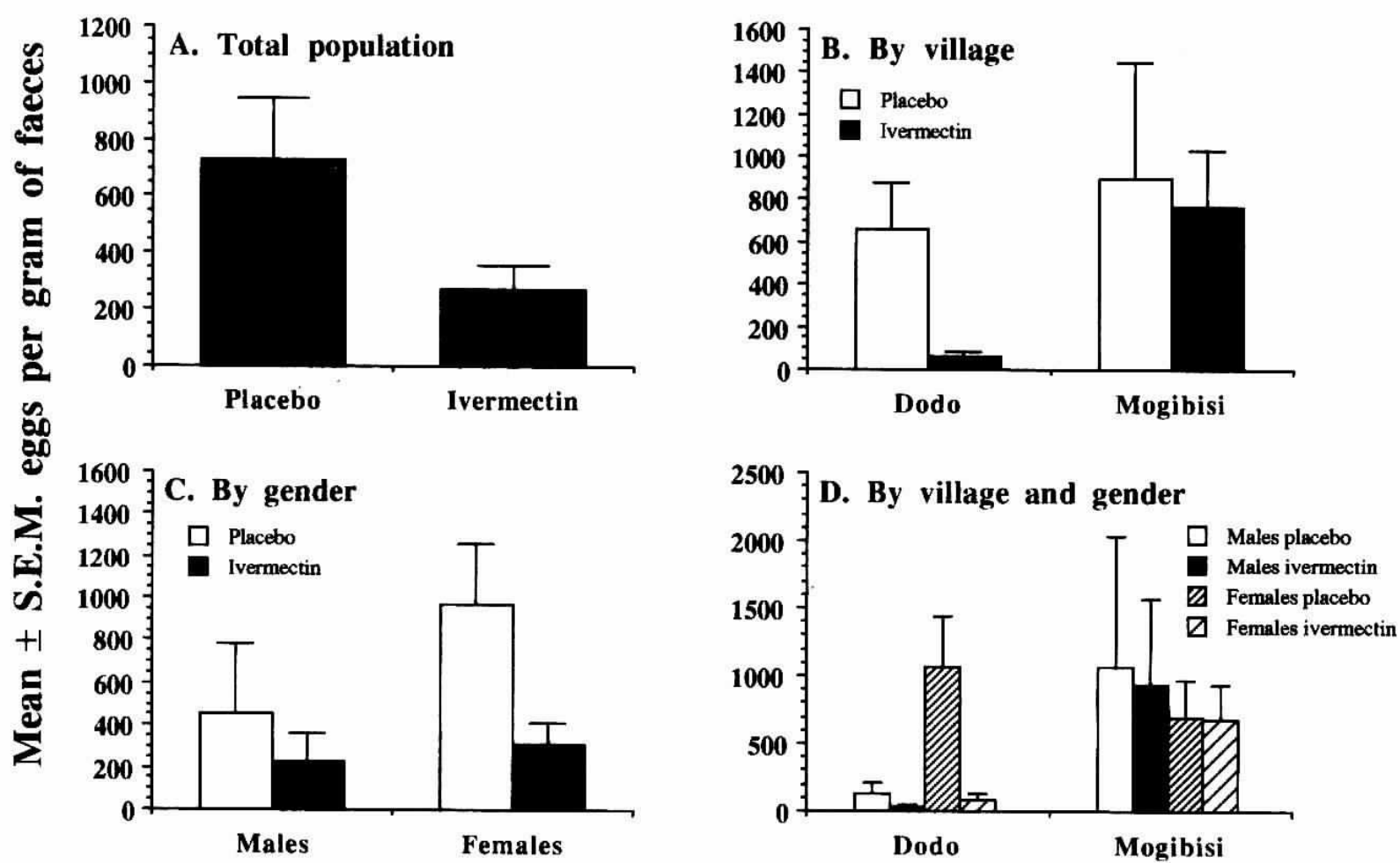

Fig. 2. Intensity of infections with Ascaris lumbricoides in ivermectin-treated and placebo-treated subjects in the total population, and by village and gender. 
among males (Dodo, 350; Mogibis, 1410), who had a lower mean EPG than females in Dodo but a markedly higher mean EPG in Mogibisi (Fig. ID). Although there was no significant effect of ivermectin on mean EPG values, these were higher among ivermectin-treated subjects in all nine comparisons with placebo treated control groups illustrated in fig. 1 .

\section{Ascaris lumbricoides}

In contrast to hookworms, the mean overall intensity of infection with $A$. lumbricoides among the placebo groups was similar in both villages (fig. 2B). However, there was a pronounced influence of gender in the combined data-set, mainly because of the high EPGs among female relative to male subjects in Dodo (the gender $\times$ village interaction in table $3, P<0.01$ ). The mean EPGs for male and female placebo-treated subjects in Dodo were 136 and 1060 respectively (fig. 2D). Overall, treatment with ivermectin reduced the mean EPG by $63.2 \%$ (fig. $2 \mathrm{~A}$ ). However, there was a discrepancy in the efficacy of treatment between the villages as reflected in the interaction between village and treatment (table 3, $P<0.01$ ). Whereas in Dodo mean Ascaris EPGs were reduced by $91.3 \%$, in Mogibisi the reduction was only $14.6 \%$. The reduction in mean EPGs in Dodo was mainly attributable to the effect of ivermectin on female subjects in which mean EPGs were reduced by $92.1 \%$ relative to the placebo group (fig. 2D). The mean EPG among male subjects in Dodo was reduced by $74.1 \%$ compared with placebo treated males, but as stated earlier the mean EPGs among the latter were much lower than among females.

\section{Trichuris trichiura}

The intensity of infection with $T$. trichiura was very low compared with the other species. There was a significant difference between the villages (fig. 3B) in the intensity of infection, but not between the sexes, mainly because males in Mogibisi had proportionally infections of higher intensity than any of the placebo-treated groups (the gender $x$ village interaction of table 3). Overall, treatment with ivermectin had a statistically significant effect despite the similar mean EPGs among ivermectin- and placebo-treated groups (fig. $3 \mathrm{~A}, \mathrm{EPGs}=12.7$ and 11.3 , respectively), but the effect was not simple because of the complex interactions of treatment $\times$ host gender and treatment $\times$ village. Thus in Mogibisi, EPGs were lower among the ivermectin-versus placebo-treated group whereas in Dodo the reverse proved to be the case. Similarly ivermectin-treated males had lower EPGs than placebo-treated males (fig. $3 \mathrm{C}$ ) whereas among female subjects, the opposite trend was observed. Overall there was no convincing significant reduction in mean $T$. trichiura EPGs: among the nine comparisons illustrated in

\section{Trichuris trichiura}
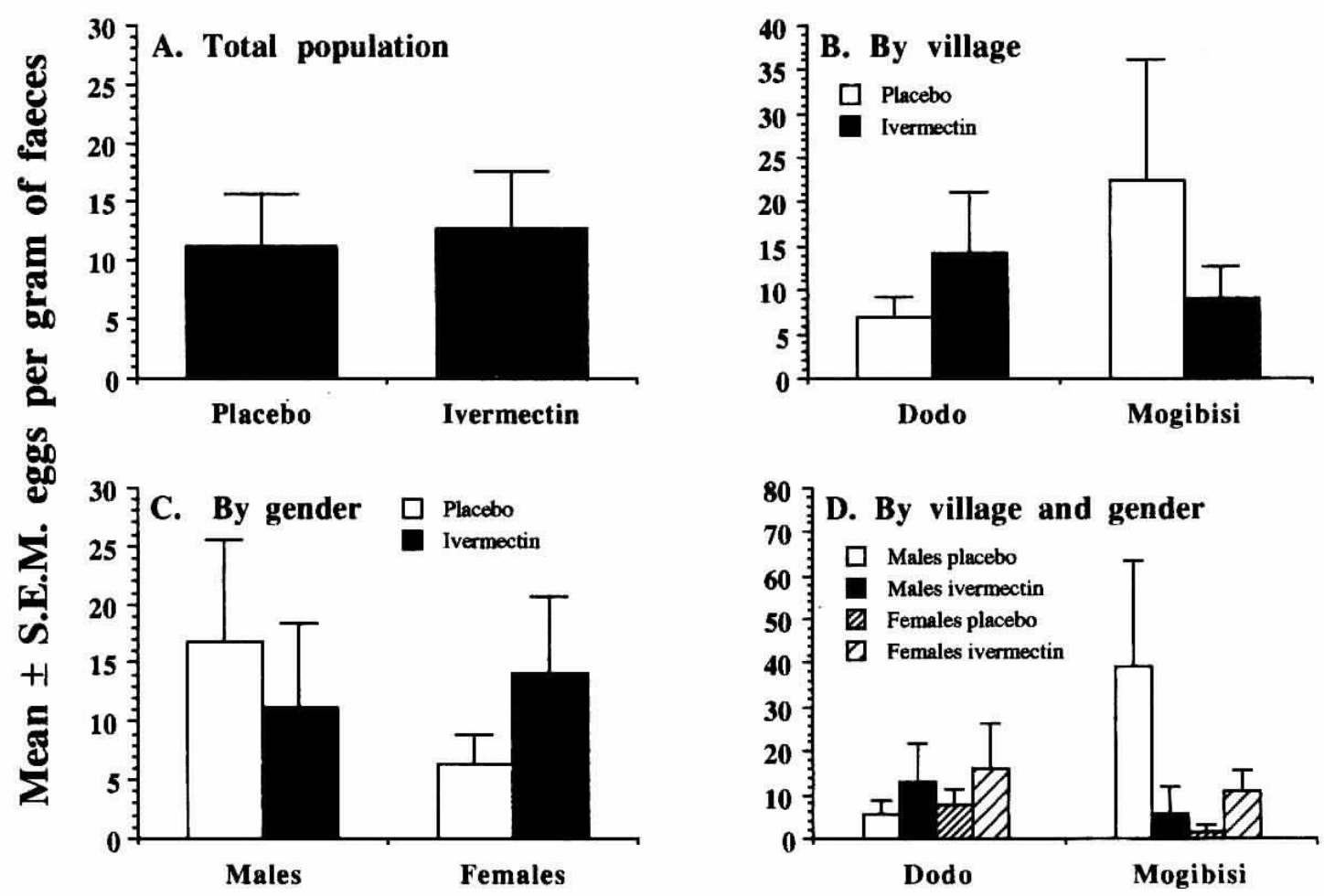

Fig. 3. Intensity of infections with Trichuris trichiura in ivermectin-treated and placebo-treated subjects in the total population, and by village and gender. 
fig. 3, six gave higher mean EPGs among ivermectin-treated subjects compared with the relevant placebo-treated control groups.

\section{Discussion}

The study reported in this paper was conducted at the end of a two year double-blind, placebo-controlled, trial of ivermectin for efficacy against onchocerciasis and, because it was not originally planned as part of the main study, it was necessarily retrospective with the placebo-treated groups providing control background information on the prevalence and intensity of infection with GI nematodes in the community. The inhabitants of six villages were offered treatment with ivermectin or placebo at six monthly intervals for four rounds in total. However, we examined the GI worm burdens 6 months after the last round of treatment only in those subjects in the two most accessible villages which had received all four rounds of treatment. Our survey was limited to these villages because of the need to complete collection of faecal samples and their laboratory analysis before all subjects, including those who had earlier received placebo treatment, were offered a final dose of ivermectin. Our sample size was also constrained by the number of inhabitants in these two villages who were interested in participating in our study although all willing subjects were examined.

In addition to comparing placebo-treated with ivermectin-treated groups directly, we chose to take village and host gender into account in the final analysis of data because the study group came from two villages of varying population size and comprised both male and female subjects. Because of the number of variables involved, we analysed the data by a novel procedure, based on the statistical package GLIM, in which the binomial distribution of the data-sets was taken into account. If, at the dose regimen used for onchocerciasis, there is to be a beneficial long-term consequence of treatment with ivermectin against GI nematodiases, it should have been clearly apparent from our data because of the sensitivity of the analytical procedures which we adopted. Instead, a complex picture was revealed with implications for other surveys of this type.

The dominant GI species in this area was N. americanus, with an overall prevalence of $90 \%$ among the placebotreated group and a mean adjusted EPG of 644. Among the various placebo-treated subsets in table 1 , the lowest prevalence recorded was $87 \%$ among females in Dodo. Ivermectin clearly had no significant effect upon either prevalence or intensity of necatoriasis. Firstly, although the prevalence was marginally lower in five of the nine comparisons between ivermectin- and placebo-treated groups in table 1 , the maximum reduction was only $8 \%$, and higher prevalence was recorded among three with no change in one case. Secondly, the mean EPGs were higher in all groups relative to controls following treatment with ivermectin, although this was not statistically significant (fig. 1). The resistance of $N$. americanus to treatment with ivermectin has been reported previously in these communities (Whitworth et al., 1991) and has been clearly demonstrated in experimental model systems (Behnke et al., 1993).

Similarly, treatment with ivermectin had no effect on the prevalence of trichuriasis; although this infection was the rarest of three GI nematodes recorded, with a maximum prevalence of $27 \%$ among the male subjects in Mogibisi but an overall prevalence among the placebo-treated subjects of $15 \%$. Our analysis yielded a significant effect of treatment on intensity of infection but this was complicated by the significant interactions between treatment $\times$ gender, treatment $\times$ village and gender $\times$ village. Essentially these interactions arose through an apparent reduction in EPG values among male subjects in Mogibisi. In all other groups ivermectin-treated subjects had higher mean EPG values relative to placebo-treated subjects (fig. 3D).

The only species which was consistently affected by ivermectin was $A$. lumbricoides. While there was little change in prevalence rates relative to the control group, there were marked changes in intensities of infections as reflected in mean EPGs (fig. 2). The group which benefited most from ivermectin were the female subjects in Dodo, where prevalence declined by $21 \%$ (table 1 ) and mean EPGs by $92.1 \%$ (fig. 2D). Males in Dodo appeared to have a lower baseline prevalence and mean EPG compared with females but nevertheless benefited significantly from treatment with a lower prevalence $(8 \%$, table 1$)$ and a reduction of mean EPGs by $74.1 \%$ (fig. 2D).

In marked contrast, ivermectin seems to have had little effect on ascariasis in Mogibisi (table 1 and fig. 2B). Initially the prevalence in the two villages was gender dependent and almost identical (males, 28, 27\%; females $50,50 \%$, Dodo and Mogibisi respectively), but unlike Dodo the prevalence among ivermectin-treated individuals in Mogibisi was either unaffected (males) or increased ( $7 \%$ females). Moreover, the mean EPGs in ivermectin- and placebo-treated groups in Mogibisi were very similar (figs 3B \& 3D). Quite clearly, ivermectin failed to show efficacy against ascariasis in Mogibisi, as assessed 6 months after four six-monthly rounds of treatment, and it is pertinent to consider likely explanations. Firstly, it is conceivable that a failure of subjects to comply with treatment might have resulted in these observations, but we think this is unlikely since all subjects took treatment in the presence of the team and were observed to swallow all doses offered. Secondly, it could have been possible for reinfection rates to be higher among a younger compared with an older population since age-intensity profiles for ascariasis in different communities show peaks in age groups around 10 (Bundy, 1990) and this has been associated with higher levels of soil-derived silica in the stools of this age group suggesting greater exposure to infection (Bundy \& Blumenthal, 1990). However, we consider differences in age to be an unlikely explanation of the higher worm burdens among males in Mogibisi because, in our study, there was little overall difference in age between the male sectors of the two villages and, in fact, the males in Mogibisi were on average older than those of Dodo (mean ages were $45.7 \pm 4.9$ and $38.1 \pm 3.2$, respectively).

An alternative explanation may be that reinfection rates were higher in Mogibisi because of generally higher contamination of the environment with Ascaris eggs, and hence worm burdens rose more rapidly in Mogibisi relative to Dodo. Thus, while prevalence with Ascaris was similar among the male placebo-treated populations of the two villages, those from Mogibisi had mean EPGs 7.8 times higher. In contrast, female subjects showed uniformly higher prevalence with Ascaris in both villages and very similar mean EPGs to the males in Mogibisi. The only real contrast therefore lies in the difference in mean intensities of 
infection among male subjects. It is also worth pointing out that males in Mogibisi had the highest intensities of infection with $T$. trichiura, which were 7.1 times higher than those among males in Dodo. It has been emphasized in the past that the distribution of $T$. trichiura and $A$. lumbricoides is often linked in communities which have both species because of their very similar modes of transmission based on long-lived eggs (Bundy, 1990) and our data support this idea. Perhaps not unexpectedly, males in Mogibisi also had the highest prevalence $(100 \%)$ and intensity of infection with $N$. americanus. Although this species is transmitted by larvae which would not survive well in the dry dusty village, they could be acquired in latrine sites in the bush on the village periphery. Collectively therefore, these data suggest that the presence of high infection intensities among male subjects in Mogibisi may have contributed to environmental contamination, and hence to higher rates of transmission relative to Dodo with all three species of GI nematodes. This may have reflected the arrangement of latrine sites and their proximity to the villages.

The results of this study confirm those of others which have shown that the wide scale use of ivermectin for the treatment of onchocerciasis, and possibly also Bancroftian filariasis in the near future, is likely to have beneficial as well as unexpected consequences for the levels of infections with GI nematodes (Whitworth et al., 1991). Whilst N. americanus and $T$. trichiura are unlikely to be affected by ivermectin chemotherapy, ascariasis may be reduced but only in the short-term. The long-term benefits of ivermectin against ascariasis will depend on the individual characteristics of local communities at the village level, with intervals between treatment and local reinfections rates (from environmental contamination by eggs) playing decisive roles in determining the outcome of treatment for particular communities.

\section{Acknowledgements}

This work was supported by MRC project grant G8928654/T. We are grateful to Dr J. Whitworth, the staff of the MRC laboratory in Bo, Sierra Leone and to the villagers of Dodo and Mogibisi for their assistance and cooperation. We wish to thank Drs G. Barnish, A. Luty and D. Chavasse for advice and logistic support, Merck Sharp and Dohme for supplying the ivermectin and placebo tablets, Pfizer for pyrantel (Combantrin) tablets, Janssen Pharmaceutials Ltd for mebendazole and $\mathrm{Dr} C$. West for supplying the GLIM macro employed in our analysis.

\section{References}

Behnke, J.M., Rose, R. \& Garside, P. (1993) Sensitivity to ivermectin and pyrantel of Ancylostoma ceylanicum and Necator americanus. International Joumal for Parasitology 23, 945-952.
Benz, G.W., Roncalli, R.A. \& Gross, S.J. (1989) Use of ivermectin in cattle, sheep, goats and swine. pp. 215-229 in Campbell, W.C. (Ed.) Ivermectin and abamectin. New York, Springer-Verlag.

Bundy, D.A.P. (1990) Is the hookworm just another geohelminth? Pp. 147-164 in Schad, G.A. \& Warren, K.S. (Eds) Hookworm disease: current status and new directions. London, Taylor \& Francis.

Bundy, D.A.P. \& Blumenthal, U.J. (1990) Human behaviour and the epidemiology of helminth infections: the role of behaviour in exposure to infection. pp. 264-289 in Barnard, C.J. \& Behnke, J.M. (Eds) Parasitism and host behaviour. London, Taylor \& Francis.

Bundy, D.A.P., Thompson, D. E., Golden, M.H.N., Cooper, E.S., Anderson, R.M. \& Harland, P.S.E. (1985) Population distribution of Trichuris trichiura in a community of Jamaican children. Transactions of the Royal Society of Tropical Medicine and Hygiene 79, 232-237.

Campbell, W.C. (1985) Ivermectin: an update. Parasitology Today 1, 10-16.

Keymer, A. \& Pagel, M. (1990) Predisposition to helminth infection. pp. 177-209 in Schad, G.A. \& Warren, K.S. (Eds) Hookworm disease: current status and new directions. London, Taylor \& Francis.

Pritchard, D.I., Quinnell, R.J., Slater, A.F.G., McKean, P.C., Dale, D.D.S., Raiko, A. \& Keymer, A.E. (1990) Epidemiology and immunology of Necator americanus infection in a community in Papua New Guinea: humoral responses to excretory-secretory and cuticular collagen antigens. Parasitology 100, 317-326.

Quinnell, R.J., Slater, A.F.G., Tighe, P.J., Walsh, E.A., Keymer, A.E. \& Pritchard, D.I. (1993) Reinfection with hookworm after chemotherapy in Papua New Guinea. Parasitology 106, 379-385.

Richard-Lenoble, D., Kombila, M., Rupp, E.A., Pappayliou, E.S., Gaxotte, P., Nguiri, C. \& Aziz, M.A. (1988) Ivermectin in loiasis and concurrent $O$. volvulus and $M$. perstans infections. American Journal of Tropical Medicine and Hygiene 39, 480-483.

Seo, B.S., Cho, S.Y., Chai, J.Y. \& Hong, S.T. (1980) Comparative efficacy of various internal mass treatment on Ascaris lumbricoides infection in Korea. Korean Journal of Parasitology 18, 145-151.

Sokal, R.R. \& Rohlf, F.J. (1981) Biometry. 2nd edn. San Francisco, Freeman \& Co.

Whitworth, J.A.G., Morgan, D., Maude, G.H., McNicholas, A.M. \& Taylor, D.W. (1991) A field study of the effect of ivermectin on intestinal helminths in man. Transactions of the Royal Society of Tropical Medicine and Hygiene 85, 232-234.

World Health Organization (1963) CCTA/WHO African conference on ancylostomiasis, Brazzaville 22-29 August 1961. Technical Report Series No. 225.

(Accepted 13 April 1994)

(C) CAB INTERNATIONAL, 1994 